\title{
Numerical Study and Analysis of Cavitation Performance in Centrifugal Impellers
}

\author{
Binama Maxime ${ }^{1}$ \\ PhD Student ${ }^{1}$ \\ Power Engineering and Engineering Thermophysics \\ Department \\ Harbin Institute of Technology
}

\begin{abstract}
Centrifugal pumps are generally used in pumping systems like water supply, sewage, and so forth. Depending on the system working conditions, centrifugal pumps can be victim to different pump failures, cavitation among others. Recent researches have been mostly oriented to the contribution of different pump parameters, be it geometrical or working parameters, to the pump performance under non-cavitating conditions. This study on the other side, intends to numerically study the contribution of the same parameters to the cavitation performance in centrifugal impellers. A numerical study was carried out with a CFD commercial code ANSYS Fluent14.5 on three impellers with different blades numbers (three, six, and nine blades) at different speeds (2120 rpm, $2560 \mathrm{rpm}$, and 3000rpm) under cavitating conditions. The pump transient flow behaviors and related fluid mass transfer were studied, where Reynolds-Averaged NavierStokes (RANS) equations were solved through the realizable k- $\mathcal{E}$ turbulence model and the Pressure Implicit with splitting of Operators (PISO) algorithm together with Schnerr and Sauer cavitation model, with water at 25 degree Celsius as the working fluid. Cavitation zones within the inter-blades flow fields constantly increased in size with both the increase in impeller blades number and rotational speed, with a thorough gradual pump head drop, leading to an almost complete inter-blades flow blockage at higher values. Low static pressure zones were merely found at each blade's suction side and gradually got wider with both parameters increase. Both parameters showed a very big effect on pump cavitation dynamics.
\end{abstract}

Keywords--Centrifugal Impeller, Cavitation, Performance Parameters, Numerical Simulations

\section{INTRODUCTION}

When the fluid pressure drops below its vapor pressure at a constant temperature, there is a thorough vapor bubbles formation in the fluid, the phenomenon which is generally referred to as "cavitation" and which generally figures among the most frequently met problems in pumping systems. In centrifugal pumps, cavitation zones are mainly found at each blades suction side at the leading edge. This is merely due to the high flow velocities, leading to subsequent pressure drop at the same places. However pump flow characteristics in general and cavitation performance in centrifugal pumps, have been shown to depend on various parameters, on which pump overall performance are based, as indicated in many already published researches. Cavitation importance is related to its effect on pump efficiency. This may be divided into three

\author{
Prof. Feng Chen-Li ${ }^{2}$ \\ Professor $^{2}$ \\ Power Engineering and Engineering Thermophysics \\ Department \\ Harbin Institute of Technology
}

categories; the modification of hydrodynamic characteristics of the flow, Damage production on boundary solid surfaces, and other extraneous effects like noise and vibration. When cavitation is ignored during the design process, it can cause catastrophic failures on the equipment leading to the whole system's efficiency drop. In the sake of the whole system safety, cavitation can be considered or controlled just during the design process of hydraulic equipment. This imposes a big limitation to the design process as it has to be very carefully controlled. In the field of fluid machinery, it has been confirmed that all turbines, from low to high speed, all pumps, be it rotodynamic or positive displacement, are susceptible to cavitation. However cavitation damages can be increased by the poor design and the faulty operating conditions of the equipment however best the design can be. With the rapid development of the computer technology and computational fluid dynamics (CFD), numerical simulation has become an important tool to study flow field in pumps and predict pump performance home and abroad. CFD analysis is a very useful tool for predicting pump performance at various operating conditions. For designers, prediction of pump operating characteristics is of a crucial importance. All theoretical methods for efficiency prediction merely give a value; but one is unable to determine the root cause for the equipment's poor performance. Due to the development of CFD code, one can get the efficiency value as well as observing the equipment actual flow conditions. Ahmed A. ${ }^{1}$ [2014] curried out a numerical simulation using CFD to investigate the effect of volute geometry on the pump performance. Simulation results were compared to the experimental data where finally the agreement between both methods results was noticed. The pump head was found to increase with pump volute cross-sectional area. Patel M.G. ${ }^{2}$ [2013] used three pumps models at different specific speeds to investigate the effect of impeller blade exit angle on centrifugal pump performance. The Gulich mathematical model was first validated with the manufacturer's H-Q curve before being used in their research. After validation, this method helped find out that both the head and pump efficiency increased with the impeller exit angle. Wei W. ${ }^{3}$ [2012] investigated the effect of inlet flow incidence angle on cavitation performance in centrifugal pumps. Based on SST k- $\omega$ turbulence model and the Mixture cavitation model, numerical simulation was carried out on four different impellers, namely R1, R2, 
R3, and R4; some with different, others with same inlet incidence angles at the blade hub, mid-span, and blade tip. The study results showed that, for a better cavitation performance, impellers should be having a large incidence angle, and that the same used incidence angle should be kept constant all along from the hub to the blade tip. An almost similar research was conducted by Baotang $\mathrm{Z}^{4}$ [2011] where, using the mixture cavitation model and the SST k- $\omega$ turbulent model, they carried out a numerical study on a double suction pump with three different impellers, namely IB1, IB2, and IB3. IB2's blade angle was smaller than IB1's by 10 degrees, whereas IB3 had 12 radial blades. The Radial impeller was found to produce higher heads but poor cavitation performance. Double suction pumps were found to produce uniform flow pattern at the upstream which could prove a better cavitation performance. Xianwu L. ${ }^{5}$ [2008], by experimentation and numerical simulation, studied the effect of the blade profile on pump cavitation performance in a miniature pump. Two semi-open impellers, the first with leaned blades the second with two-dimensional blades, were studied; where $\mathrm{k}-\varepsilon$ turbulence model and VOF cavitation model were used for the simulation process. The leaned blades were found preferential to both the hydraulic and cavitation performance; however, the increase in axial tip clearance was found to make the pump cavitation performance even worse. Xianwu L. ${ }^{6}$ [2008] again curried out an experimental and numerical research on the influence of impeller inlet geometry on pump cavitation performance. Five different impellers were studied where the blade inlet angle and blade leading edge were being gradually extended. The numerical simulation was conducted based on RNG $\mathrm{k}-\mathcal{E}$ turbulence model and the VOF cavitation model. The pump inlet geometry proved to be influential when it comes to centrifugal pump performance, where larger blade leading edge and blade inlet angle positively affected the pump hydraulic performance and cavitation performance respectively. The present study intends to use CFD to predict cavitation performance through variation of centrifugal impeller geometry (blades number) and pump working conditions (Pump speed).

\section{MATHEMATICAL MODEL}

A mathematical model can be defined as the combination of dependent and independent variables and relative parameters in the form of a set of differential equations which defines and governs the physical phenomenon [7]. In this study, bubble formation and cavitation zones development was investigated using Computational Fluid Dynamics (CFD) modeling in three different impellers as shown in figure 2. The ANSYS FLUENT 14.5's Schnerr and Sauer Cavitation Model were used together with the mixture multiphase model. These models solve the multiphase flow equations, with mass transfer due to cavitation as the source and sink terms in liquid and vapor continuity equations where the mixture multiphase model treats the two phases as interpenetrating. The flow turbulence was solved by the Realizable K-E turbulence model along with PISO algorithm.

\section{A. Governing Equations}

The mixture continuity equation is presented by the next equation:

$$
\frac{\partial\left(\rho_{\mathrm{m}}\right)}{\partial \mathrm{t}}+\nabla \cdot\left(\rho_{\mathrm{m}} \overrightarrow{\mathrm{u}}_{\mathrm{m}}\right)=0
$$

The mixture momentum equation was calculated by summing all phase's individual momentum equations to end with:

$$
\begin{aligned}
& \frac{\partial}{\partial t}\left(\rho_{m} \vec{u}_{m}\right)+\nabla \cdot\left(\rho_{m} \vec{u}_{m} \vec{u}_{m}\right)= \\
& -\nabla p+\nabla \cdot\left[\mu_{m}\left(\nabla \vec{u}_{m}+\nabla \vec{u}_{m}^{T}\right)\right]+ \\
& \rho_{m} \vec{g}+\vec{F}
\end{aligned}
$$

Where $\nabla \cdot\left(\rho_{\mathrm{m}} \overrightarrow{\mathrm{u}}_{\mathrm{m}} \overrightarrow{\mathrm{u}}_{\mathrm{m}}\right), \quad \frac{\partial}{\partial \mathrm{t}} \rho_{\mathrm{m}} \mathrm{u}_{\mathrm{m}}$, and $\quad\left[\mu_{\mathrm{m}}\left(\nabla \overrightarrow{\mathrm{u}}_{\mathrm{m}}+\right.\right.$ $\left.\left.\nabla \overrightarrow{\mathrm{u}}_{\mathrm{m}}^{\mathrm{T}}\right)\right]$ stand for kinetic energy, inertia, and viscous forces. $\mu_{m}$ and $\vec{F}$ stand for mixture viscosity and body force. The second phase's volume fraction is deducted from its continuity equation as:

$$
\begin{aligned}
& \frac{\partial}{\partial \mathrm{t}}\left(\alpha_{\mathrm{p}} \rho_{\mathrm{p}}\right)+\nabla \cdot\left(\alpha_{\mathrm{p}} \rho_{\mathrm{p}} \overrightarrow{\mathrm{u}}_{\mathrm{m}}\right)= \\
& \nabla \cdot\left(\alpha_{\mathrm{p}} \rho_{\mathrm{p}} \overrightarrow{\mathrm{u}}_{\mathrm{dr}, \mathrm{p}}\right)+ \\
& \sum_{\mathrm{q}=1}^{\mathrm{n}}\left(\mathrm{m}_{\mathrm{qp}}-\mathrm{m}_{\mathrm{pq}}\right)
\end{aligned}
$$

B. The Realizable $k$-Eturbulence model

This model is an improved Standard k- $\varepsilon$ model.

In this model two Partial differential Equations are developed; one for the turbulent kinetic energy $\mathrm{k}$, another for the turbulence dissipation rate $\mathcal{E}$. Turbulence Kinetic Energy is expressed by:

$$
\begin{aligned}
\frac{\partial \rho \mathrm{K}}{\partial \tau}+\frac{\partial \rho \mathrm{KU}_{\mathrm{j}}}{\partial \mathrm{x}_{\mathrm{j}}}= & \\
\frac{\partial}{\partial \mathrm{x}_{\mathrm{j}}}\left[\left(\mu+\frac{\mu_{\mathrm{t}}}{\sigma_{\mathrm{k}}}\right)\right. & \left.\frac{\partial_{\mathrm{k}}}{\partial \mathrm{x}_{\mathrm{j}}}\right]+\mathrm{P}_{\mathrm{k}}+\mathrm{G}_{\mathrm{k}} \\
& -\rho_{\varepsilon}
\end{aligned}
$$

The $\mathrm{k}$ dissipation rate is:

$$
\begin{aligned}
& \frac{\partial \rho \varepsilon}{\partial \tau}+\frac{\partial \rho \varepsilon U_{j}}{\partial \mathrm{x}_{\mathrm{j}}}= \\
& \frac{\partial}{\partial \mathrm{x}_{\mathrm{j}}}\left[\left(\mu+\frac{\mu_{\mathrm{t}}}{\sigma_{\varepsilon}}\right) \frac{\partial \varepsilon}{\partial \mathrm{x}_{\mathrm{j}}}\right]+\mathrm{C}_{\varepsilon 1} \\
& \left(\left(\mathrm{P}_{\mathrm{k}}\right)+\mathrm{C}_{\varepsilon 3} \mathrm{G}_{\mathrm{k}}\right) \frac{\varepsilon}{\mathrm{K}}-\mathrm{C}_{\varepsilon 3} \rho \frac{\varepsilon^{2}}{\mathrm{~K}}
\end{aligned}
$$

Where the turbulent viscosity is given by:

$$
\mu_{\mathrm{t}} \equiv \rho \mathrm{C}_{\mu} \frac{\mathrm{k}^{2}}{\varepsilon}
$$

In the above presented equations, $G_{K}$ represents turbulence kinetic energy generation. 


\section{Schnerr and Sauer Cavitation model}

Cavitation is the fluid rupture phenomenon from the fluid pressure drop under its vapor pressure, accompanied by the mass transfer between the liquid and the gas phases.

It is governed by Rayleigh-Plesset equation:

$$
\begin{aligned}
& \Re_{\mathrm{B}} \frac{\mathrm{D}^{2} \mathfrak{R}_{\mathrm{B}}}{\mathrm{Dt} \mathrm{t}^{2}}+\frac{3}{2}\left(\frac{\mathrm{D} \mathfrak{R}_{\mathrm{B}}}{\mathrm{Dt}}\right)^{2}= \\
& \left(\frac{\mathrm{P}_{\mathrm{B}}-\mathrm{P}}{\mathrm{P}_{\ell}}\right)-\frac{4 \mathrm{~V}_{\ell}}{\mathfrak{R}_{\mathrm{B}}} \Re_{\mathrm{B}}-\frac{2 \mathrm{~S}}{\rho_{\ell} \Re_{\mathrm{B}}}
\end{aligned}
$$

In this model, the vapor volume fraction has the following expression:

$$
\begin{aligned}
& \frac{\partial}{\partial \mathrm{t}}\left(\propto \rho_{\mathrm{V}}\right)+\nabla\left(\alpha \rho_{\mathrm{V}} \overrightarrow{\mathrm{V}}\right)= \\
& \frac{\rho_{\ell} \rho_{\mathrm{V}}}{\rho} \frac{\mathrm{D} \alpha}{\mathrm{Dt}}
\end{aligned}
$$

The general expression connecting the mass sources $\mathrm{R}$ to gas bubble radius is given by:

$$
\mathrm{R}=\frac{\rho_{\mathrm{v}} \rho_{\ell}}{\rho} \alpha(1-\alpha) \frac{3}{\mathfrak{R}_{\mathrm{B}}} \sqrt{\frac{2}{3} \frac{\mathrm{P}_{\mathrm{v}}-\mathrm{P}}{\rho_{\ell}}}
$$

The above transfer rate (R) equation is used for condensation process modeling; here down is the final model:

When $\mathrm{P}_{\mathrm{v}} \geq \mathrm{P}$ :

$$
\begin{array}{r}
\mathrm{R}_{e}=\frac{\rho_{\mathrm{v}} \rho_{\ell}}{\rho} \alpha(1-\alpha) \\
\frac{3}{\Re_{\mathrm{B}}} \sqrt{\frac{2}{3} \frac{\mathrm{P}_{\mathrm{v}}-\mathrm{P}}{\rho_{\ell}}}
\end{array}
$$

When $\mathrm{P}_{\mathrm{v}} \leq \mathrm{P}$ :

$$
\begin{array}{r}
\mathrm{R}_{c}=\frac{\rho_{\mathrm{v}} \rho_{\ell}}{\rho} \alpha(1-\alpha) \\
\frac{3}{\Re_{\mathrm{B}}} \sqrt{\frac{2}{3} \frac{\mathrm{P}-\mathrm{P}_{\mathrm{v}}}{\rho_{\ell}}}
\end{array}
$$

Where $\Re_{B}, \sigma, \rho_{\ell}, \mathrm{P}_{\mathrm{B}}, \mathrm{P}$ stand for bubble radius, liquid surface tension coefficient, liquid density, bubble surface pressure, and local far-field pressure respectively.

\section{III.GEOMETRIC MODEL}

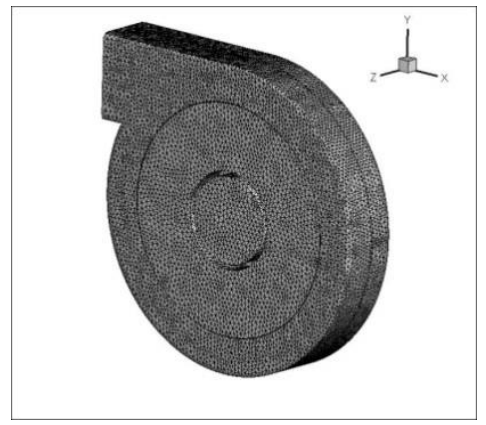

Figure1. Pump computational grid.
Three domains were built according the written pump structural design theory, using professional geometric modeling software called SOLIDWORKS 2013. The required mesh was created by ANSYS ICEM CFD software.

For all the three domains, the impeller inlet and outlet diameter was $48 \mathrm{~mm}$ and $122 \mathrm{~mm}$ respectively. All used casings have the same dimensions whereas their impellers differ in the number of blades per each (3, 6, and 9 blades).
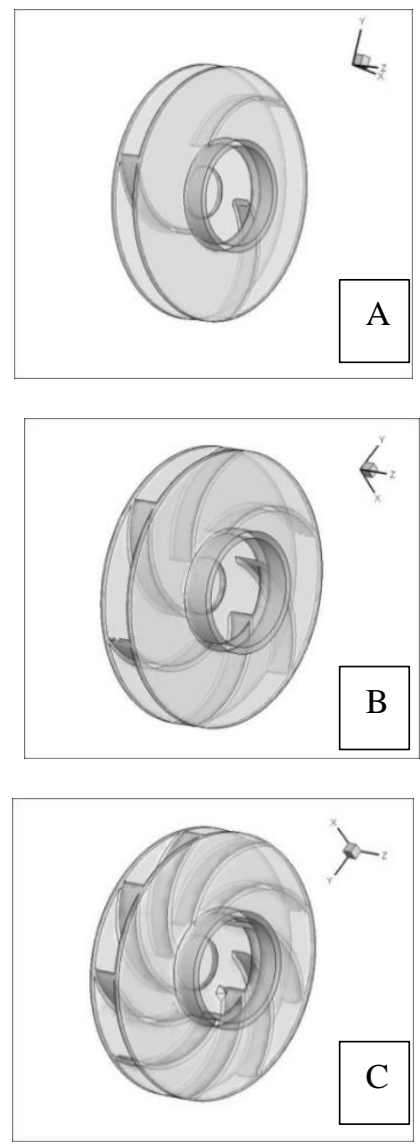

Figure2. Geometric models of the three used impellers [A: 3 blades impeller, B: 6 blades impeller, and C: 9 blades impeller]

Table 1: Model geometric specifications

\begin{tabular}{ll}
\hline Parameter & Value \\
Blade Shape & Circular Arc \\
Blade Thickness & $3 \mathrm{~mm}$ \\
Blade Inlet Width & $12 \mathrm{~mm}$ \\
Blade Outlet Width & $12 \mathrm{~mm}$ \\
Blades Number & 3,6, and 9 \\
Impeller Type & Closed \\
\hline
\end{tabular}

\section{NUMERICAL SIMULATIONS AND RESULTS}

In this study, the working fluid is a mixture of water at 25 degree Celsius and water vapor. The gas phase velocity at the pump inlet zone is zero under the assumption that the flow is transient. The Schnerr and Sauer cavitation model was used for mass transfer between the liquid and gas phases. The mixture governing equations were solved by commercial a CFD code ANSYS Fluent coupled with its Realizable k-E turbulence model through a widely known 
PISO discretization algorithm. The momentum and turbulence convection terms equations were discretized by using the quick scheme.

\section{A. Boundary conditions}

The boundary conditions were the constant flow velocity at inlet and the outlet pressure. The non-slip boundary conditions were also imposed on the pump side walls as well as on the impeller blades. The rotating and stationary reference frames were set at the rotating impeller and remaining stationary zones respectively, and are coupled by a multi rotating frame with an interface between them to take care of their interactions.

\section{B. Pump flow simulation results and analysis \\ a. Pump Static pressure distribution}

As shown in figure 3 , the static pressure in every impeller increases from the blade leading edge toward the trailing edge, but that only happens on the blade pressure side. Low pressure zones are predominantly found on every blade suction side, and grow with both the blades number and impeller rotational speed.

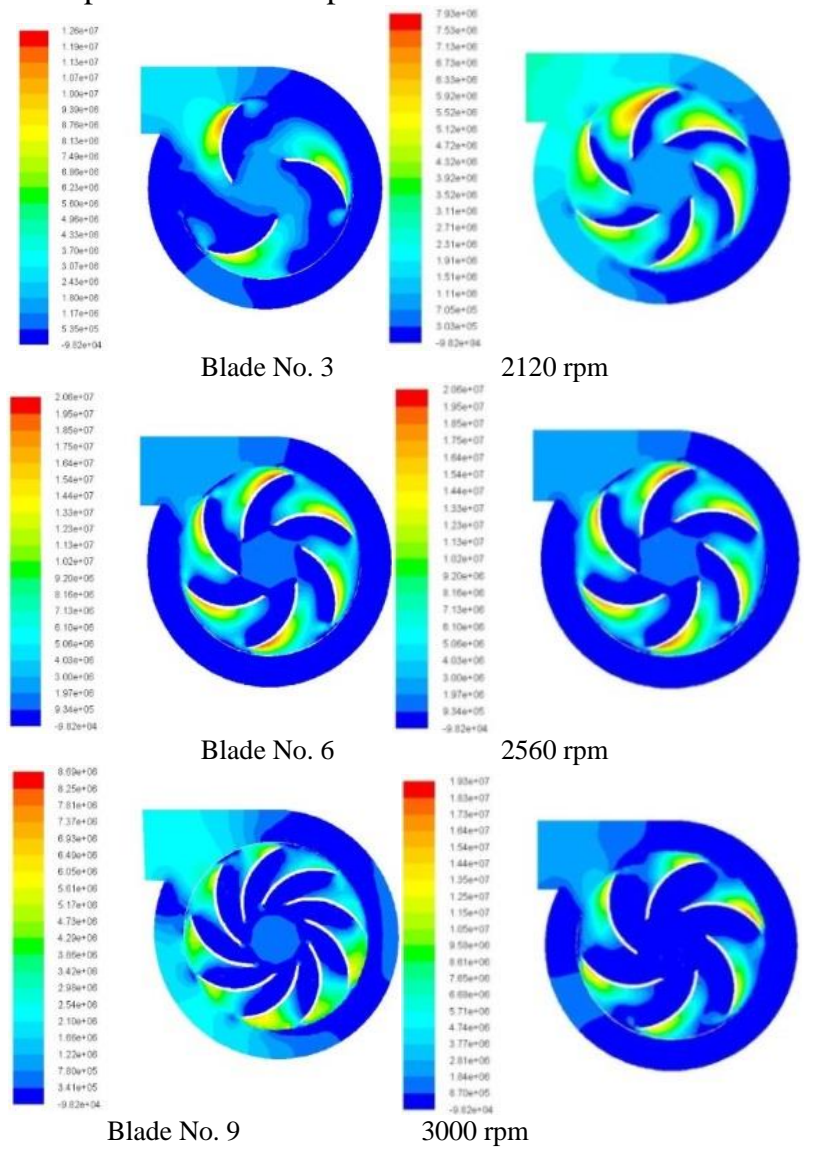

Figure3. Static pressure distribution in the mid span for different impellers at different speeds

The static pressure at the pump inlet gets smaller and smaller whereas it gradually increases when approaching the pump outlet zones, with the increase in both parameters.
The maximum pressure value increased with the blades number from 3 to 6 blades with $1.26 \mathrm{e}+07$ and $2.6 \mathrm{e}+7$ Pascal respectively. The nine blades impeller, due to the flow constriction at the inter-blade flow areas, showed a worse performance in terms of both pressure and developed head with 8.69e+6 Pascal and $162.6 \mathrm{~m}$ respectively as shown in figure4.

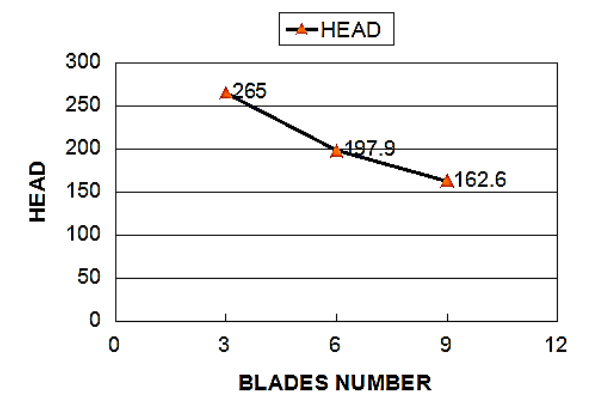

Figure4. Developed head with different blade numbers

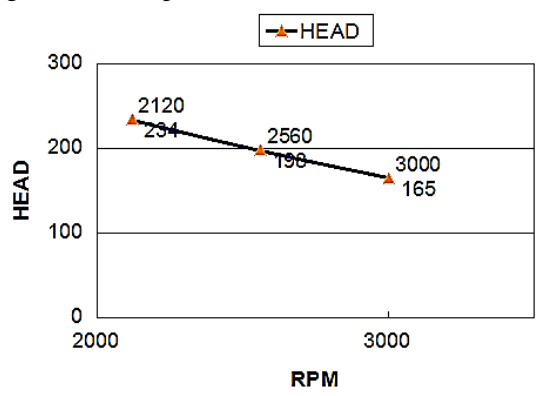

Figure5. Developed Head at different speeds

For the parameter impeller speed, Maximum static pressure was found to change depending of the rotational speed where the 2560 RPM impeller rotation speed was found to deliver a higher maximum Static pressure with 2.06e+07 Pascal. A thorough continuous pump head drop was also noticed from $234 \mathrm{~m}$ to $165 \mathrm{~m}$.

\section{b. Pump cavitation performance}
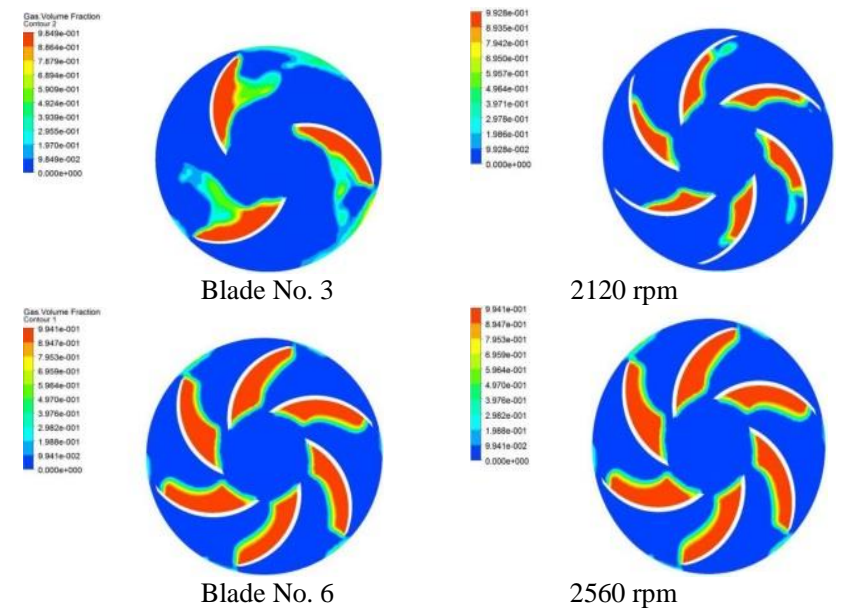

$2560 \mathrm{rpm}$ 

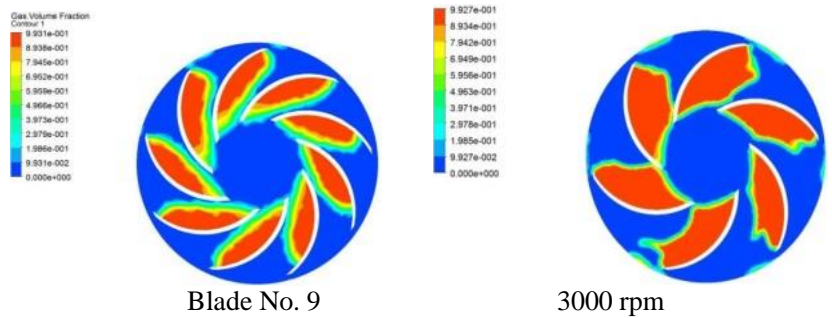

Figure6. Gas volume fraction evolution with different impellers and at different speeds .

The above figure 6 also clearly shows the increase of cavitation region sizes with both the increase in blades number and impeller rotational speed, almost blocking the flow at higher values, and resulting in the continuous pump Head drop.

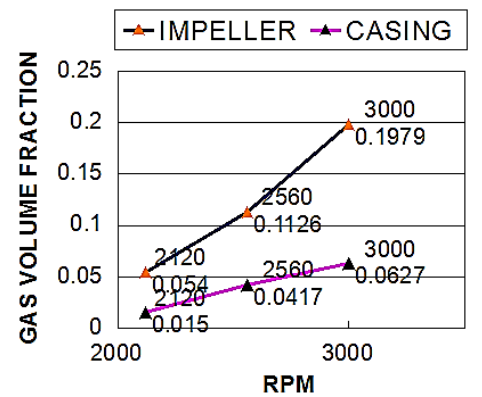

Figure7. Gas phase volume fraction at different speeds

Cavitation phenomena has been found to occur at the impeller flow zones more than at pump casing flow zones. Gas phase volume fraction has been noticed to gradually grow at both the impeller and casing flow areas with the impeller rotational speed increase.

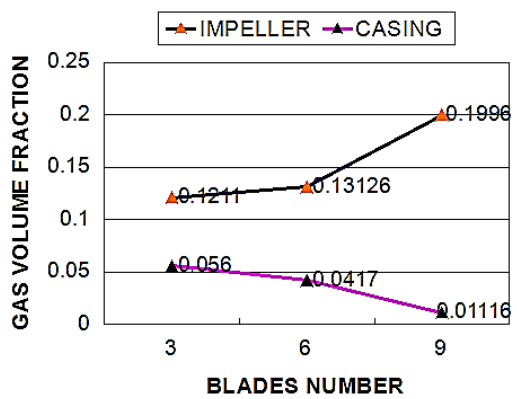

Figure8. Gas phase volume fraction at different blades number

\section{CONCLUSION}

The study of both the pump design (Impeller Blades number) and working (Impeller rotational speed) parameters effect on pump cavitation performance was carried out using Ansys Fluent Software. High pressure zones were generally found at the blade pressure side's trailing edge whereas low pressure ones were merely located at the blade suction side's leading edge. With the increase in blades number, the inter-blade flow field got gradually reduced thus increasing the flow velocity at these areas and a subsequent pressure drop, giving rise to the cavitation phenomenon and a thorough gradual Head drop. With increase in the impeller rotational speed, the flow velocity at the impeller flow areas got higher and higher, thus increasing the cavitation intensity in the pump. The static pressure at the pump inlet continually reduced with the increase in both parameters. The pump head also gradually reduced with the increase in both parameters while the change in static pressure with the variation of both the blades number and impeller speed is a little bit complex.

\section{REFERENCES}

[1] Al-Obaidi, Ahmed, Suman Pradhan, Taimoor Asim, Rakesh Mishra, and Karina Zala. Numerical studies of the velocity distribution within the volute of a centrifugal pump.(2014).

[2] Patel, M. G., and A. V. Doshi. "Effect of Impeller Blade Exit Angle on the Performance of Centrifugal Pump." Int J. Emerging Technology and Advanced Engineering 3, no. 1 (2013): 91-99.

[3] Wei, W., X. W. Luo, B. Ji, B. T. Zhuang, and H. Y. Xu. Cavitating flow investigation inside centrifugal impellers for a condensate pump, IOP Conference Series: Earth and Environmental Science, vol. 15, no. 3, p. 032061. IOP Publishing, 2012.

[4] Zhuang, Baotang, Xianwu Luo, Lei Zhu, Xin Wang, and Hongyuan $\mathrm{Xu}$. Cavitation in a shaft-less double suction centrifugal miniature pump., International Journal of Fluid Machinery and Systems 4, no. 1 (2011): 191-198.

[5] Luo, Xianwu, Shuhong Liu, Yao Zhang, and Hongyuan Xu., Cavitation in semi-open centrifugal impellers for a miniature pump, Frontiers of Energy and Power Engineering in China 2, no. 1 (2008): 31-35.

[6] Luo, Xianwu, Yao Zhang, Junqi Peng, Hongyuan Xu, and Weiping Yu., Impeller inlet geometry effect on performance improvement for centrifugal pumps, Journal of Mechanical Science and Technology 22, no. 10 (2008): 1971-1976.

[7] Pandey, K. M., A. P. Singh, and Sujoy Chakraborty. "Numerical studies on effects of blade number variations on performance of centrifugal pumps AT $2500 \mathrm{rpm}$." Journal of Environmental Research And Development 6, no. 3A (2012).

On the other side, as shown in the above figure, due to the flow recirculation phenomenon occurrence in the three blades impeller, but which gradually decreased due to the blades number increase, the gas phase volume fraction in the pump casing flow zones has been decreasing with the increase in impeller blades number. 